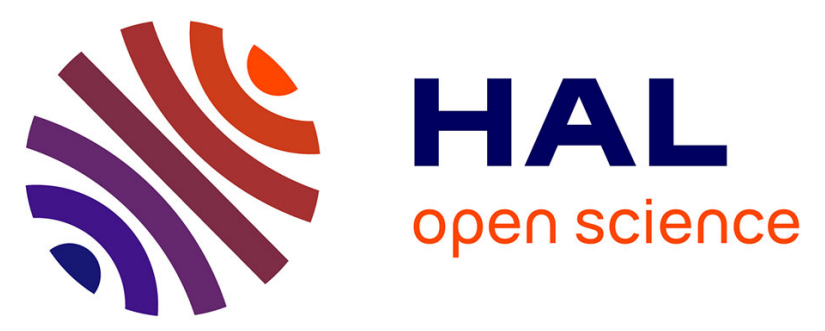

\title{
A comparison between Nonlinear Least Squares and Maximum Likelihood estimation for the prediction of tumor growth on experimental data of human and rat origin
}

Spyridon Patmanidis, Roberto Chignola, Alexandros C. Charalampidis, George P. Papavassilopoulos

\section{To cite this version:}

Spyridon Patmanidis, Roberto Chignola, Alexandros C. Charalampidis, George P. Papavassilopoulos. A comparison between Nonlinear Least Squares and Maximum Likelihood estimation for the prediction of tumor growth on experimental data of human and rat origin. Biomedical Signal Processing and Control, 2019, 54, pp.101639. 10.1016/j.bspc.2019.101639 . hal-02283074

\section{HAL Id: hal-02283074 \\ https://hal.science/hal-02283074}

Submitted on 11 Sep 2019

HAL is a multi-disciplinary open access archive for the deposit and dissemination of scientific research documents, whether they are published or not. The documents may come from teaching and research institutions in France or abroad, or from public or private research centers.
L'archive ouverte pluridisciplinaire HAL, est destinée au dépôt et à la diffusion de documents scientifiques de niveau recherche, publiés ou non, émanant des établissements d'enseignement et de recherche français ou étrangers, des laboratoires publics ou privés. 


\title{
A Comparison between Nonlinear Least Squares and Maximum Likelihood Estimation for the Prediction of Tumor Growth on Experimental Data of Human and Rat Origin
}

\author{
Spyridon Patmanidis ${ }^{\mathrm{a}, *}$, Roberto Chignola ${ }^{\mathrm{b}}$, Alexandros C. Charalampidis ${ }^{\mathrm{c}, \mathrm{d}}$, \\ George P. Papavassilopoulos ${ }^{\mathrm{a}}$ \\ ${ }^{a}$ School of Electrical and Computer Engineering, National Technical University of Athens, \\ Iroon Polytechneiou 9, Zografou 15780, Athens, Greece \\ ${ }^{b}$ Department of Biotechnology, University of Verona, Cà Vignal 1, Strada Le Grazie 15, \\ 37134, Verona, Italy \\ ${ }^{c}$ Department of Electrical Engineering and Computer Science, Technische Universität \\ Berlin, Control Systems Group, Einsteinufer 17, Berlin D-10587, Germany \\ ${ }^{d}$ CentraleSupélec, Automatic Control Group - IETR, Avenue de la Boulaie, 35576 \\ Cesson-Sévigné, France
}

\section{Abstract}

Several mathematical models have been developed to explain the growth of tumors and used to fit experimental or clinical data. Their predictive power i.e. their ability to forecast the future growth on the basis of present knowledge - however, has been rarely explored. Here, we investigate whether a Hidden Markov Model (HMM) based on the well-established Gompertz tumor growth function with additive Gaussian noise could effectively be used to predict the future growth of experimental tumors. The idea behind this work is that one might achieve more accurate predictions if estimates of the unknown parameters of the HMM are used instead of those obtained by fits of the deterministic Gompertz model to the data. We use the principle of Maximum Likelihood (ML) to estimate unknown parameters related to growth dynamics and noise,

\footnotetext{
* Corresponding author: Spyridon Patmanidis. Address: ECE Building, Office: 21.42, Iroon Polytechneiou 9, Zografou 15780, Athens, Greece Tel: +302107722545 , Mob: +306972818983

Email addresses: spatmanid@gmail.com (Spyridon Patmanidis), roberto.chignola@univr.it (Roberto Chignola), alexandros.charalampidis@centralesupelec.fr (Alexandros C. Charalampidis), yorgos@netmode.ece.ntua.gr (George P. Papavassilopoulos)
} 
and we compare its effectiveness to the classical Nonlinear Least Squares minimization approach. The analyses show that our approach can provide better growth predictions when the data contain adequate information concerning the tumors saturation phase. The forecasts could also be improved by taking into account prior knowledge about the unknown parameters when the information concerning the saturation phase was inadequate. We conclude that by using HMMs in combination with the principle of ML, one can obtain more reliable growth predictions for individual tumors.

Keywords: Tumor Growth, Nonlinear Systems, Parameter Estimation, Maximum Likelihood, Nonlinear Least Squares, Hidden Markov Model, Noise.

\section{Introduction}

Human cancers are usually treated with one or a combination of therapies such as chemotherapy, immunotherapy, radiotherapy, and surgery. In order to improve the therapeutic outcome, the use of mathematical models along with

5 optimal control has been proposed by many researchers [1]. Michor and Beal [5] present extensive literature on the topic of how mathematical modeling can be applied to deliver better drug regimes. In this context, the importance of mathematical modeling is apparent since the models' ability to describe, and eventually predict, tumor growth greatly affects the outcome of the therapy. However, there is no general consensus about which could be the best mathematical model of tumor growth.

Part of the problem is the complexity of the tumor biology. For example, in the case of solid tumors cancer and normal cells actively communicate by means of a number of molecular signals and conspire to shape the tumor microenvironment and finally to regulate the overall growth of the tumor itself. The molecular network includes positive and negative feedbacks and its nonlinear character challenges mathematical modeling. Models that attempt to capture this microscopic complexity have been developed, but their use in the clinical settings is limited owed to their high computational costs 6 . For this reason, 
simple phenomenological models developed in the past are still used to describe tumor growth. The limited number of observations that can be collected in the clinical settings, however, does not allow to obtain enough data to compare the accuracy of different growth models. For example, whether the growth of real tumors is limited above or not is still a matter of debate [7]. As a further complication, it has been recently shown that even in the case of blood tumors, where it is much easier to draw tumor cells from patients and study their growth kinetics, some clones appear to grow exponentially while others show sigmoidal growth up to a steady-state level [8].

Experimental solid tumors can provide data to test mathematical models, 30 and in this case it has been shown that the Gompertz model is a good analytical descriptor of the macroscopic features of tumor growth and of biological growth in general 9 913 . It is a phenomenological model that describes exponential growth with an exponentially decreasing growth rate, and it has also been shown to approximate quite reasonably the behavior of biologically-motivated, but

35 more complex, tumor growth models [14. Its mathematical simplicity but at the same time its ability to capture the macroscopic features of experimental tumors make the Gompertz model a good choice to test new forecasting approaches.

In our previous works, we proposed and tested with synthetic data a methodology that estimates the unknown parameters of the Gompertz model [15 17].

40 The first one is called growth rate and is related to the cells ability to proliferate, while the second one is called carrying capacity and is related to the tumors saturation volume. In the literature based to the findings of Brunton and Wheldon [11 13, the carrying capacity is usually considered as a fixed species-specific parameter when the Gompertz model is used to fit curves to experimental data

45 [18 20, and a Nonlinear Least Squares estimator is used to estimate the growth rate. This approach usually provides curves that fit well to the data, especially when the number of available measurements is large and the tumors have reached a growth maturity. Yet, it ignores the observed heterogeneity of tumor growth that has been recognized to have clinical implications in the planning 50 of effective treatment schedules 21,24 . This heterogeneity is likely to stem 
from the peculiar microscopic characteristics of each tumor, features that at the macroscopic level translate into growth behaviors, and thus Gompertz growth parameters, that vary between individual tumors [21 24].

Taking that heterogeneity into consideration, we proposed a Hidden Markov Model (HMM) based on the Gompertz function that considers both the carrying capacity and the growth rate as patient-specified parameters. In order to estimate the unknown parameters of the HMM, we used the principle of Maximum Likelihood [25]. The properties of Maximum Likelihood Estimators (MLEs) have been extensively in the past and can be also found in [25]. The MLE implementation was presented in detail in [17] and was able to estimate the growth parameters of the Gompertz model with accuracy on synthetic tumor data. The difficulty of working with HMMs lies in the inability to measure the hidden states. However, the proposed MLE algorithm can successfully deal with this challenge and provide accurate estimates of the unknown parameters.

65 Some other challenges are the uncertainty which arises due to the small size of the datasets and the calibration that the algorithm needs in order to provide accurate estimates [16, 17].

In this work, experimental data from past works are being used [14, 19, 26, 27]. The data are time series of volume size measured for individual multicell 70 tumor spheroids (MTSs) in vitro. MTSs are three-dimensional aggregates of tumor cells that can be obtained and cultured in vitro under controlled experimental conditions [28]. They represent a tumor model with an intermediate complexity between standard two-dimensional monolayer cultures in vitro and in vivo tumors, as they approximate many biological characteristics of small non-vascularized tumors or of intervascular regions of larger tumors [28]. These include - but are not limited to - the heterogeneous expression of cell surface markers, the production of an intercellular matrix, the heterogeneous distribution of nutrients and of waste molecules and the presence of a central core of quiescent and eventually dead cells [28. MTSs show a neat spherical morphology and this fact is commonly exploited to convert their diameters, measured at the microscope or by by image analysis, into spheroid volumes [14, 26 28]. In 
turn, this also means that we can summarize the microscopic three-dimensional complexity of the spheroids in just one measured parameter and thus use a simple one-dimensional model to describe their growth kinetics. In particular, the growth kinetics of MTSs are well described by the Gompertz model [14, 24, 26, 27, and thus MTSs are an excellent experimental system to test the accuracy of new forecasting methods based on the Gompertz growth law.

The data have been obtained with different cell types from human and rat tumors, and thus they can allow to take into account the species-specific character of the Gompertz model discussed above. In particular we analyze the time-dependent volume growth of individual spheroids obtained with human breast carcinoma (MCF7) and glioblastoma - a brain cancer - (U118) cells and with rat glioblastoma tumors (9L) [19]. Along with the MLE from our previous works and the commonly used NLS approach, we implemented two variations for the NLS approach. In the first one, we consider the carrying capacity as an unknown parameter to be estimated. In the second one, instead of fixing the carrying capacity to the maximum attained value (MAV) from the available dataset, we fixed the carrying capacity to the mean value of the mean of the last $\mathrm{n}$ measurements of each dataset (mentioned as Mean of Means - MM). This work aims to answer two main questions: 1 . whether the proposed model can be used effectively to describe and predict the tumor growth of experimental data and 2. how this approach performs if compared to the standard nonlinear fit of the Gompertz model to growth data.

In the following sections, we present the materials and describe the methods (Section 2), present the simulation results (Section 3), explain our findings (Section 4) and discuss future work Section 5).

\section{Materials and Methods}

\subsection{Data}

The MTSs were obtained using U118 (human glioblastoma), 9L (rat glioblastoma) [19, 26, 27] and MCF7 (human breast carcinoma) cell lines [14]. Overall, 
32 9L spheroids, 8 U118 spheroids, and 5 MCF7 spheroids were considered for growth analysis. A detailed description about how the cancer cells were cultured to obtain spheroids can be found in the aforementioned references [14, 19, 26, 27]. Some differences between these data sets, however, deserve to spheroids was measured from calibrated micrographs taken with a digital microscope (EVOSci, AMG, Bothell, WA, USA) by using the image analysis software ImageJ (http://imagej.nih.gov). Starting from single cells a time period of $\sim 60$ days was appropriate to accurately determine the first quasi-exponential growth growth kinetics. On average the MCF7 dataset comprises $24.8 \pm 8.6$ volume 
measurements/spheroid (min. 15, max. 33).

\subsection{Tumor Growth Modeling}

The stochastic discrete time state-space representation of the Gompertz model, which was introduced in [16] and also used in [17, was used here to describe the growth dynamics. This model can be formulated as follows:

$$
\begin{aligned}
x_{k+1} & =f\left(x_{k}, \theta_{1}, \theta_{2}\right)+w_{k}, \\
y_{k} & =x_{k}+v_{k},
\end{aligned}
$$

where $x_{k}\left(\mathrm{~mm}^{3}\right)$ is the tumor volume at time step $k, y_{k}$ the a measurement that corresponds to the state $x_{k}$, and $k \in \mathbb{N}$. The function $f$ in Eq. (1a) is the state-space representation of the Gompertz function [30]:

$$
f\left(x_{k}, \theta_{1}, \theta_{2}\right)=\theta_{2} \exp \left(\ln \left(\frac{x_{k}}{\theta_{2}}\right) \exp \left(-\frac{1}{\theta_{1}} t_{k+1}\right)\right),
$$

where $\theta_{1}$ (days) is related to the cells' proliferative ability, $\theta_{2}\left(\mathrm{~mm}^{3}\right)$ is the carrying capacity $\left(\lim _{k \rightarrow \infty} x_{k}=\theta_{2}\right)$ and $t_{k+1}$ (days) is the time between $k$ and $k+1$. The random variables $w_{k}$ and $v_{k}, k \in \mathbb{N}$ are mutually independent and normally distributed with zero mean and unknown variance: $w_{k} \sim \mathcal{N}\left(0, \sigma_{w_{k}}^{2}\right)$ and $v_{k} \sim \mathcal{N}\left(0, \sigma_{v_{k}}^{2}\right)$, where $\sigma_{w_{k}}=\theta_{3} x_{k}^{\theta_{4}}$ and $\sigma_{v_{k}}=\theta_{5} x_{k}^{\theta_{6}}$. Measurements are available from time $\mathrm{k}=1$ onwards. Because of the random components $w_{k}$ and $v_{k}$, the model of Eq. (1) also corresponds to the description:

$$
\begin{aligned}
x_{k+1} & \sim p_{\boldsymbol{\theta}_{\boldsymbol{a}}}\left(x_{k+1} \mid x_{k}\right), \\
y_{k} & \sim p_{\boldsymbol{\theta}_{\boldsymbol{b}}}\left(y_{k} \mid x_{k}\right),
\end{aligned}
$$

where $p_{\theta_{a}}\left(x_{k+1} \mid x_{k}\right)$ is the probability density function describing the dynamics for given values of $x_{k}$ and $p_{\theta_{b}}\left(y_{k} \mid x_{k}\right)$ is the probability density function describing the measurements [31, 32]. In Eq. (3a) $\boldsymbol{\theta}_{\boldsymbol{a}}=\left[\theta_{1}, \theta_{2}, \theta_{3}, \theta_{4}\right]^{T}$, where $\boldsymbol{\theta}_{\boldsymbol{a}} \in \Theta_{a}$ with $\Theta_{a} \subseteq \mathbb{R}_{>0}^{4}$ denoting a compact set of permissible values of the unknown vector $\boldsymbol{\theta}_{\boldsymbol{a}}$ and in Eq. (3b) $\boldsymbol{\theta}_{\boldsymbol{b}}=\left[\theta_{5}, \theta_{6}\right]^{T}$, where $\boldsymbol{\theta}_{\boldsymbol{b}} \in \Theta_{b}$ with $\Theta_{b} \subseteq \mathbb{R}_{>0}^{2}$ denoting a compact set of permissible values of the unknown vector $\boldsymbol{\theta}_{\boldsymbol{b}}$. We also define the vector $\boldsymbol{\theta}=\left[\boldsymbol{\theta}_{\boldsymbol{a}}, \boldsymbol{\theta}_{\boldsymbol{b}}\right]$, where $\boldsymbol{\theta} \in \Theta$ with $\Theta \subseteq \mathbb{R}_{>0}^{6}$. 


\subsection{Parameter Estimation}

The problem addressed in this manuscript is how to obtain an estimate $\hat{\boldsymbol{\theta}}$ based on $N$ measurements $\boldsymbol{Y}_{N}=\left[y_{1}, \ldots, y_{N}\right]$ and predict the future states $\hat{\boldsymbol{X}}_{N+1: M}=\left[\hat{x}_{N+1}, \ldots, \hat{x}_{M}\right]$. In order to estimate the unknown parameters im-

plemented methods based on the Nonlinear Least Squares and the Maximum Likelihood. In this manuscript, we include a brief description of the Maximum Likelihood estimator.

\subsubsection{Nonlinear Least Squares Estimator}

This method is used to find the estimates $\hat{\theta}_{1}$ and $\hat{\theta}_{2}$ of the parameters $\theta_{1}$ and $\theta_{2}$ that minimize the summation of the squared difference between the measurements $\boldsymbol{Y}_{n}$ and the estimates $\hat{\boldsymbol{X}}_{N}$ :

$$
\left[\hat{\theta}_{1}, \hat{\theta}_{2}\right]=\underset{\theta_{1} \in \Theta_{1}, \theta_{2} \in \Theta_{2}}{\arg \min } \sum_{1}^{N}\left(y_{k}-\hat{x}_{k}\right)^{2} .
$$

where $\theta_{1} \in \Theta_{1}$ with $\Theta_{1} \subseteq \mathbb{R}_{>0}$ and $\theta_{2} \in \Theta_{2}$ with $\Theta_{2} \subseteq \mathbb{R}_{>0}$ denote compact sets of permissible values for the unknown parameters $\theta_{1}$ and $\theta_{2}$, and $x_{k}$ is given by:

$$
\hat{x}_{k}=\theta_{2} \exp \left(\ln \left(\frac{\hat{x}_{k-1}}{\theta_{2}}\right) \exp \left(-\frac{1}{\theta_{1}} t_{k}\right)\right) .
$$

The modifications needed in order to test the cases in which the carrying capacity is fixed to the MAV or MM are trivial.

\subsubsection{Maximum Likelihood Estimator}

This method is applied to the model of Eq. (1) and is used to find an estimate $\hat{\boldsymbol{\theta}}$ of the vector $\boldsymbol{\theta}$ that maximizes the joint density $p_{\boldsymbol{\theta}}\left(\boldsymbol{Y}_{N}\right)$ of the observation:

$$
\hat{\boldsymbol{\theta}}=\underset{\boldsymbol{\theta} \in \Theta}{\arg \max } p_{\boldsymbol{\theta}}\left(\boldsymbol{Y}_{N}\right)
$$

Bayes' rule can be used in order to decompose the joint density according to

$$
p_{\boldsymbol{\theta}}\left(\boldsymbol{Y}_{N}\right)=p_{\boldsymbol{\theta}}\left(y_{1}\right) \prod_{k=2}^{N} p_{\boldsymbol{\theta}}\left(y_{k} \mid \boldsymbol{Y}_{k-1}\right)
$$

where

$$
p_{\boldsymbol{\theta}}\left(y_{k+1} \mid \boldsymbol{Y}_{k}\right)=\int p_{\boldsymbol{\theta}}\left(y_{k+1} \mid x_{k+1}\right) p_{\boldsymbol{\theta}}\left(x_{k+1} \mid \boldsymbol{Y}_{k}\right) d x_{k+1},
$$




$$
p_{\boldsymbol{\theta}}\left(x_{k+1} \mid \boldsymbol{Y}_{k}\right)=\int p_{\boldsymbol{\theta}}\left(x_{k+1} \mid x_{k}\right) p_{\boldsymbol{\theta}}\left(x_{k} \mid \boldsymbol{Y}_{k}\right) d x_{k},
$$

and

$$
p_{\boldsymbol{\theta}}\left(x_{k+1} \mid \boldsymbol{Y}_{k+1}\right)=\frac{p_{\boldsymbol{\theta}}\left(y_{k+1} \mid x_{k+1}\right) p_{\boldsymbol{\theta}}\left(x_{k+1} \mid \boldsymbol{Y}_{k}\right)}{p_{\theta}\left(y_{k+1} \mid \boldsymbol{Y}_{k}\right)} .
$$

The distributions in Eqs. (8) to (10) can be computed iteratively. However, in most cases, as also in the case studied in this manuscript, the above integrals cannot be solved analytically. Nevertheless, since $x_{k}$ is low-dimensional, numerical integration can be used effectively in order to compute the integrals of Eqs. (9) and (10). More specifically, we divide the integration interval into subintervals and we compute each subintergral by using the trapezoidal rule. The summation of these subintegrals provides an approximation of the initial integral we want to compute. Moreover, to reduce the interval of integration $\left[0, X_{\max }\right]$ the Gaussian property of the noise is being used. At every iteration step, $x_{k}$ is considered a normally distributed random variable with mean $\mu=y_{k}$ and variance $\sigma^{2}=\left(\sigma_{0} y_{k}^{e_{0}}\right)^{2}\left(x_{k} \sim \mathcal{N}\left(\mu, \sigma^{2}\right)\right)$. As a result, the interval $[\mu-5 \sigma$, $\mu+5 \sigma] \bigcap\left[0, X_{\max }\right]$, which is significantly smaller than $\left[0, X_{\max }\right]$, can be used as the interval of integration [17. For a more detailed description of the Maximum Likelihood Estimator, the reader may refer to Section 3.1.3 Numerical Maximum Likelihood in [17.

\section{Results}

In this section, we present the simulation results. In order to perform these simulations we used MATLAB (version R2015a, The MathWorks Inc., Natick, IL). The computational methods were implemented by writing suitable functions, while Eqs. (4) and (6) were computed with the fmincon function [33].

In this work, we examined three different datasets that contain multicell tumor spheroids. MCF7 refers to human breast carcinoma cell line, U118 to human glioblastoma cell line and 9L to rat glioblastoma cell line. Fig. (1) shows plots of the growth data for all datasets in order to inform the reader on the type of experimental data we used for the simulations. We implemented 
the ML estimator proposed in [17] and a Least Squares estimator and compared their performance. For the ML estimator, both the growth rate and the carrying capacity were estimated at each time step. As regards the LS estimator, along with the approach proposed in the literature where the carrying capacity is fixed to a certain known value, we also examined the case in which this parameter is considered unknown (refered below as $\mathrm{LS}_{2 p}$ ). In the case that the carrying capacity is fixed, two sub-cases were studied. In the first one, the MAV of each dataset was considered as the fixed value of carrying capacity (referred below as $\mathrm{LS}_{M A V}$ ), while in the second one the MM (referred below as $\mathrm{LS}_{M M}$ ). The value of MM is given by:

$$
\mathrm{MM}=\frac{1}{n M} \sum_{i=1}^{M} \sum_{j=N-n}^{N} y_{i, j},
$$

where $n$ is the number of measurements used to obtain the mean carrying capacity of the spheroids, $M$ is the number of spheroids the dataset contains, $i$ is the spheroid's ID and $j$ is the measurement's ID. In our simulations, $n$ has been set to 10 for the U118 and the 9L datasets, while for the MCF7 dataset it has been set to 5 due to the small number of available measurements. The parameter estimates were used in order to predict the tumors' future growth.

The Root-Mean-Square Deviation (RMSD) was used in order to measure the accuracy of the predictions. Given $Y_{N}=\left[y_{1}, \cdots, y_{N}\right]$, the first $k$ measurements $\left(Y_{1: k}=\left[y_{1}, \cdots, y_{k}\right], k<N\right)$ were used to estimate the growth dynamics $\hat{\theta}_{1}$ and $\hat{\theta}_{2}$. The future growth of the tumor $\left(\hat{X}_{k+1: N}=\left[\hat{x}_{k+1}, \cdots, \hat{x}_{N}\right]\right)$ was predicted by the following equation:

$$
\hat{x}_{i}=\hat{\theta}_{2} \exp \left(\ln \left(\frac{\hat{x}_{i-1}}{\hat{\theta}_{2}}\right) \exp \left(-\frac{1}{\hat{\theta}_{1}} t_{i}\right)\right),
$$

where $i=2, \cdots, N$ and $\hat{x}_{1}=y_{1}$. Then, the RMSD was computed by:

$$
\operatorname{RMSD}=\sqrt{\frac{\sum_{i=k+1}^{N}\left(y_{i}-\hat{x}_{i}\right)^{2}}{N-(k+1)}}
$$

The RMSD of the LS estimator will be referred to as RMSD $_{L S}$ and the RMSD of the ML estimator as $\mathrm{RMSD}_{M L}$. 
Table 1: Number of tumors for which the RMSD of the predictions is lower for the Maximum Likelihood approach. The Maximum Likelihood approach is compared to each Least Squares approach seperately. This table refers to the MCF7 dataset.

\section{MCF7}

No. of Available Measurements

\begin{tabular}{|c|c|c|c|c|c|c|c|c|c|c|c|c|}
\hline & 2 & 3 & 4 & 5 & 6 & 7 & 8 & 9 & 10 & 11 & 12 & 13 \\
\hline \multicolumn{13}{|c|}{ ML vs $\operatorname{LS}_{M A V}$} \\
\hline No. of Tumors & 1 & 4 & 2 & 1 & 1 & 1 & 0 & 0 & 0 & 1 & 0 & 0 \\
\hline \multicolumn{13}{|c|}{ ML vs $\mathrm{LS}_{M M}$} \\
\hline No. of Tumors & 2 & 3 & 4 & 3 & 3 & 2 & 2 & 2 & 2 & 2 & 1 & 1 \\
\hline \multicolumn{13}{|c|}{ ML vs $\mathrm{LS}_{2 p}$} \\
\hline No. of Tumors & 2 & 3 & 3 & 2 & 2 & 2 & 1 & 0 & 1 & 1 & 0 & 0 \\
\hline
\end{tabular}
was lower than $\operatorname{RMSD}_{L S}$ (cases where the ML performed better). The ML method is compared to each Least Squares approach for each dataset seperately. We also present the comparison between the mean RMSD of the prediction at each time step of ML and $\mathrm{LS}_{M A V}$ in Figs. (2), of ML and $\mathrm{LS}_{M M}$ in Figs. (3). and of ML and $\mathrm{LS}_{2 p}$ in Figs. (4),

\section{Discussion}

Even though the ability of a broad range of mathematical models (including Gompertz) to describe experimental data is studied in several studies, the predictive power is rarely considered, as Benzekry et al. point in [7. This work examines whether an HMM based on the Gompertz function with additive Gaussian noise can be used effectively in order to describe and predict the growth of experimental tumor data. This model entails the challenge of computing the prediction density $p_{\theta}\left(y_{k} \mid Y_{k-1}\right)$ in absence of the hidden states $x_{k}$. Our main hypothesis is that we can achieve more accurate predictions for the future growth of a tumor if we use the estimates of the unknown parameters of the 
Table 2: Number of tumors for which the RMSD of the predictions is lower for the Maximum Likelihood approach. The Maximum Likelihood approach is compared to each Least Squares approach seperately. This table refers to the U118 dataset.

\section{$\mathrm{U} 118$}

\section{No. of Available Measurements}

\begin{tabular}{|c|c|c|c|c|c|c|c|c|c|c|}
\hline & 2 & 3 & 4 & 5 & 6 & 7 & 8 & 9 & 10 & 11 \\
\hline \multicolumn{11}{|c|}{$\mathrm{ML}$ vs $\mathrm{LS}_{M A V}$} \\
\hline No. of Tumors & 5 & 2 & 3 & 3 & 3 & 2 & 2 & 4 & 4 & 5 \\
\hline \multicolumn{11}{|c|}{$\mathrm{ML}$ vs $\mathrm{LS}_{M M}$} \\
\hline No. of Tumors & 4 & 4 & 4 & 3 & 4 & 3 & 3 & 2 & 7 & 6 \\
\hline \multicolumn{11}{|c|}{$\mathrm{ML}$ vs $\mathrm{LS}_{2 p}$} \\
\hline No. of Tumors & 5 & 7 & 6 & 6 & 3 & 3 & 3 & 5 & 5 & 6 \\
\hline
\end{tabular}

No. of Available Measurements

\begin{tabular}{llllllllll}
12 & 13 & 14 & 15 & 16 & 17 & 18 & 19 & 20 \\
\hline
\end{tabular}

ML vs $\mathrm{LS}_{M A V}$

\begin{tabular}{cccccccccc} 
No. of Tumors & 5 & 5 & 5 & 5 & 3 & 5 & 4 & 5 & 3 \\
\hline ML vs $\operatorname{LS}_{M M}$
\end{tabular}

No. of Tumors $\quad 4 \quad 4 \quad \begin{array}{llllllll}5 & 5 & 4 & 4 & 4 & 5 & 4\end{array}$

ML vs $\mathrm{LS}_{2 p}$

No. of Tumors $\quad 4 \quad 4 \quad 4 \quad 5 \quad \begin{array}{lllllll}5 & 3 & 5 & 3 & 3 & 1\end{array}$ 
Table 3: Number of tumors for which the RMSD of the predictions is lower for the Maximum Likelihood approach. The Maximum Likelihood approach is compared to each Least Squares approach seperately. This table refers to the $9 \mathrm{~L}$ dataset.

$9 \mathrm{~L}$

\begin{tabular}{|c|c|c|c|c|c|c|c|c|c|c|}
\hline & \multicolumn{10}{|c|}{ No. of Available Measurements } \\
\hline & 2 & 3 & 4 & 5 & 6 & 7 & 8 & 9 & 10 & 11 \\
\hline \multicolumn{11}{|c|}{ ML vs $\mathrm{LS}_{M A V}$} \\
\hline No. of Tumors & 31 & 29 & 29 & 32 & 32 & 31 & 30 & 30 & 31 & 30 \\
\hline \multicolumn{11}{|c|}{ ML vs $\mathrm{LS}_{M M}$} \\
\hline No. of Tumors & 4 & 9 & 7 & 7 & 5 & 5 & 5 & 6 & 9 & 8 \\
\hline \multicolumn{11}{|c|}{ ML vs $\mathrm{LS}_{2 p}$} \\
\hline No. of Tumors & 13 & 16 & 18 & 18 & 16 & 22 & 17 & 17 & 16 & 16 \\
\hline
\end{tabular}

No. of Available Measurements

$\begin{array}{llllllllll}12 & 13 & 14 & 15 & 16 & 17 & 18 & 19 & 20 & 21\end{array}$

ML vs $\mathrm{LS}_{M A V}$

$\begin{array}{lllllllllll}\text { No. of Tumors } & 27 & 27 & 28 & 26 & 27 & 27 & 27 & 25 & 27 & 29\end{array}$

ML vs $\mathrm{LS}_{M M}$

\begin{tabular}{lllllllllll} 
No. of Tumors & 10 & 14 & 16 & 16 & 16 & 15 & 16 & 17 & 22 & 23 \\
\hline
\end{tabular}

ML vs $\mathrm{LS}_{2 p}$

\begin{tabular}{lllllllllll} 
No. of Tumors & 16 & 16 & 12 & 13 & 12 & 12 & 8 & 13 & 18 & 19 \\
\hline
\end{tabular}

No. of Available Measurements

$\begin{array}{lllllllll}22 & 23 & 24 & 25 & 26 & 27 & 28 & 29 & 30\end{array}$

ML vs $\mathrm{LS}_{M A V}$

$\begin{array}{llllllllll}\text { No. of Tumors } & 30 & 29 & 29 & 29 & 30 & 31 & 31 & 30 & 32\end{array}$

ML vs $\operatorname{LS}_{M M}$

$\begin{array}{llllllllll}\text { No. of Tumors } & 25 & 23 & 25 & 21 & 24 & 23 & 24 & 23 & 24\end{array}$

ML vs $\mathrm{LS}_{2 p}$

$\begin{array}{llllllllll}\text { No. of Tumors } & 20 & 14 & 16 & 18 & 23 & 23 & 22 & 19 & 23\end{array}$ 
HMM instead of the estimates obtained by the classical approach of the deterministic Gompertz model with the carrying capacity fixed to a species-specific parameter. We also hypothesize that the growth predictions can be improved by utilizing prior knowledge about the unknown parameters. In order to test closer look at Figs. (1a) reveals that until the 13th measurement (which is the time window when we try to estimate the growth rate and the carrying capacity in order to predict the future growth) the spheroids are far from the saturation phase, which means that there is not enough information considering 235 cells, i.e. the data are from tumor spheroids that started their growth when they 
were formed by one cell only, with the aim to investigate in details the initial quasi-exponential growth phase. The plateau phase is not well characterized (see also Fig 1) and this is a critical point since the ML estimates the carrying capacity directly from the data. Further research with a larger dataset that monitors the growth for a longer time interval data could lead to better results.

In regard to the U118 dataset, we can see a different behavior. The NLS approach with the carrying capacity fixed to the MAV performs better than MLE at the early growth stages (when there are only a few available measurements). As the growth progressed, the MLE approach provides more accurate predictions in the majority of cases, even though the mean $\mathrm{RMSD}_{L S}$ is slightly lower than the mean $\operatorname{RMSD}_{M L}$. This behavior is expected since the saturation phase for this dataset has been reached during the examined time window. When the carrying capacity is unknown, the performance of NLS drops and MLE performs generally better for both the early and later growth stages. Lastly, using the MM as carrying capacity has a positive influence at the early and negative at later growth stages.

9L spheroids were monitored for a longer period of time (thus there are more samples for each spheroid, see Section 2.1) and the saturation phase was reached more quickly. As a result, for this dataset, the MLE over-performs the NLS approach. By considering the carrying capacity unknown and to be estimated, the performance of the NLS estimator improved, but the ML estimator still performed slightly better. However, using the MM as carrying capacity greatly improved the NLS prediction ability at early stages. At later growth stages, the MLE is again able to provide more accurate predictions.

Overall, our analyses indicate that the monitoring time interval is a decisive factor for the prediction of the future growth. Whether or not the data contain enough information for the saturation phase is therefore critical to make good predictions of tumor growth with the Gompertz model. When the data lack this information, the approaches that fix the carrying capacity are expected to have better performance since the fixed parameter includes some information about the saturation phase. However, even though the approaches with the fixed 
parameter may provide curves that fit well to the data, they are in many cases unable to predict the tumors future growth, because the information about the saturation phase might be inaccurate (due to the intrinsic growth variability of individual tumor spheroids even when they are produced with cells from the same cell line [24].

As regards our first hypothesis, we confirmed that the proposed HMM is able to describe the growth of experimental tumors. Furthermore, we observed that when a tumor starts to grow rapidly and the measurements contain information about the saturation phase, this approach provided, in most cases, more accurate predictions of future growth. This is however a potential problem since, as far as we know, there are no firm data concerning the saturation phase of in vivo solid tumors because in general they are treated a short time after their diagnosis. It is tempting to speculate that each organ can nonetheless contain tumors of a given maximum size and it might be interesting to infer the carrying capacity of different organs on the basis of some biophysical characteristics, like e.g. their biomechanical properties, or from statistical analyses of clinical cancer data such as tumor size at patients' death. For example, it is well known that solid-stress from the surrounding environment can inhibit the growth of experimental tumors and affect the plateau phase of their growth kinetics [38. Tissue-specific estimates might then be used as prior knowledge to inform and possibly validate our approach also for in vivo tumors. Indeed, we show that the utilization of prior knowledge in the NLS estimator can improve the growth predictions at early growth stages, but as the tumor grows, we can obtain estimates that are able to predict tumor growth more accurately by combining the HMM model with the MLE.

At this point, we would like to notice some ideas for further research that might be of interest. First of all, it would be very interesing to examine the descriptive power of models other than the Gompertz. To put it in another way, instead of the stochastic model of Eq. (1), the MLE can be combined with an HMM (based on another growth law) that can describe certain cancer tumors better than the Gompertz. This could lead to a more accurate growth 
prediction for particular types of tumors. Another interesting topic for further

Maximum Likelihood with the prior knowledge about the unknown parameters.

\section{Conclusions}

In our previous works, we proposed a new approach for the estimation of the Gompertz's model parameters and tested it on synthetic tumor data. In this

315

its potential to predict the future growth of a tumor.

The main contribution is that this work shows the ability of the proposed HMM to describe the tumor growth of experimental data. Furthermore, it indicates that the proposed approach can be used for tumor growth prediction and phase of the tumor, it can provide more accurate tumor growth predictions compared to the classical approach. Lastly, it shows that the utilization of the prior knowledge concerning the unknown parameters has the potential to improve the growth predictions at early growth stages where the data may lack information

To conclude, it should be noted that our estimation technique is not limited to the Gompertz model, and thus the MLE can be applied to other models 
that may describe tumor growth models more appropriately (e.g. [14]). We believe that individualized tumor growth modeling is of great importance since it takes into consideration the heterogeneous growth of individual tumors and thus it has the potential to provide more accurate results. The MLE is a general approach that can be exploited for this purpose.

\section{Conflict of interest}

The authors of this manuscript declare that they have no financial and personal relationship with other people or organizations that could inappropriately influence their work.

\section{Acknowledgment}

Spyridon Patmanidis wishes to thank the Special Account for Research of the National Technical University of Athens for supporting his doctoral research through a scholarship of excellence.

The work of Alexandros Charalampidis has been supported by the European Union's Horizon 2020 research and innovation programme under the Marie Skłodowska-Curie grant agreement No 705982.

\section{References}

[1] M. M. Hadjiandreou, G. D. Mitsis, Mathematical modeling of tumor growth, drug-resistance, toxicity, and optimal therapy design, IEEE Transactions on Biomedical Engineering 61 (2) (2014) 415-425. doi:10.1109/ TBME. 2013.2280189

[2] C. Babbs, Predicting success or failure of immunotherapy for cancer: insights from a clinically applicable mathematical model, American Journal of Cancer Research 2 (2) (2012) 204-213. 
[3] R. Serre, S. Benzekry, L. Padovani, C. Meille, N. André, J. Ciccolini, F. Barlesi, X. Muracciole, D. Barbolosi, Mathematical modeling of cancer immunotherapy and its synergy with radiotherapy, Cancer Research 76 (17) (2016) 4931-4940. doi:10.1158/0008-5472.CAN-15-3567.

[4] S. Benzekry, A. Tracz, M. Mastri, R. Corbelli, S. Barbolosi, J. M. L. Ebos, Modeling spontaneous metastasis following surgery: An in vivo-in silico approach, Cancer Research 76 (3) (2016) 535-547. doi:10.1158/0008-5472. CAN-15-1389.

[5] F. Michor, K. Beal, Improving cancer treatment via mathematical modeling: Surmounting the challenges is worth the effort, Cell 163 (5) (2015) 1059-1063. doi:http://dx.doi.org/10.1016/j.cell.2015.11.002.

[6] J. Metzcar, Y. Wang, R. Heiland, P. Macklin, A review of cell-based computational modeling in cancer biology, JCO Clinical Cancer Informatics 3 (2019) 1-13. doi:10.1200/CCI.18.00069.

[7] S. Benzekry, C. Lamont, A. Beheshti, A. Tracz, J. M. L. Ebos, L. Hlatky, P. Hahnfeldt, Classical mathematical models for description and prediction of experimental tumor growth, PLOS Computational Biology 10 (8) (2014) 1-19. doi:10.1371/journal.pcbi.1003800

[8] M. Gruber, I. Bozic, I. Leschiner, D. Livitz, K. Stevenson, L. Rassenti, D. Rosebrock, A. Taylor-Wiener, O. Olive, R. Goyetche, S. M. Fernandes, J. Sun, C. Stewart, C. C. A. Wong, W. Zhang, J. G. Reiter, J. M. Gerold, J. G. Gribben, K. R. Rai, M. J. Keating, J. R. Brown, D. Neuberg, T. J. Kipps, M. A. Nowak, G. Getz, C. J. Wu, Growth dynamics in naturally 375 ㅁ progressing chronic lymphocytic leukemia, Nature (2019) doi:https:// doi.org/10.1038/s41586-019-1252-x.

[9] A. K. Laird, Dynamics of tumor growth, British Journal of Cancer 18 (1964) 490-502. 
[10] A. K. Laird, Dynamics of growth in tumors and normal organisms, National Cancer Institute Monograph 30 (1969) 15-28.

[11] G. F. Brunton, T. E. Wheldon, Prediction of the complete growth pattern of human multiple myeloma from restricted initial measurements, Cell Proliferation 10 (6) (1977) 591-594. doi:10.1111/j.1365-2184.1977. tb00316.x.

[12] G. F. Brunton, T. E. Wheldon, Characteristic species dependent growth patterns of mammalian neoplasms, Cell Proliferation 11 (2) (1978) 161-175. doi:10.1111/j.1365-2184.1978.tb00884.x

[13] G. F. Brunton, T. E. Wheldon, The gompertz equation and the construction of tumour growth curves, Cell Proliferation 13 (4) (1980) 455-460. doi:10.1111/j.1365-2184.1980.tb00486.x

[14] E. Milotti, V. Vladislav, M. Sega, R. Chignola, Interplay between distribution of live cells and growth dynamics of solid tumours, Scientific Reports 2 (990) (2012) 1-7. doi:http://dx.doi.org/10.1038/srep00990.

[15] B. Gompertz, On the nature of the function expressive of the law of human mortality, and on a new mode of determining the value of life contingencies, Philosophical Transactions of the Royal Society of London 115 (1825) 513583.

[16] S. Patmanidis, A. C. Charalampidis, I. Kordonis, G. D. Mitsis, G. P. Papavassilopoulos, Comparing methods for parameter estimation of the gompertz tumor growth model, IFAC-PapersOnLine 50 (1) (2017) 12203-12209. doi:https://doi.org/10.1016/j.ifacol.2017.08.2289

[17] S. Patmanidis, A. C. Charalampidis, I. Kordonis, G. D. Mitsis, G. P. Papavassilopoulos, Tumor growth modeling: Parameter estimation with maximum likelihood methods, Computer Methods and Programs in Biomedicine 160 (2018) 1-10. doi:https://doi.org/10.1016/j.cmpb.2018.03.014. 
[18] C. Loizides, D. Iacovides, M. M. Hadjiandreou, G. Rizki, A. Achilleos, K. Strati, G. D. Mitsis, Model-based tumor growth dynamics and therapy response in a mouse model of de novo carcinogenesis, PLOS ONE 10 (12) (2015) 1-18. doi:10.1371/journal.pone.0143840

[23] H. Bartelink, A. Begg, J. C. Martin, M. van Dijk, L. van 't Veer, P. van der Vaart, M. Verheij, Towards prediction and modulation of treatment re-

[24] R. Chignola, R. Foroni, A. Franceschi, M. Pasti, C. Candiani, C. Anselmi, G. Fracasso, G. Tridente, M. Colombatti, Heterogeneous response of individual multicellular tumour spheroids to immunotoxins and ricin toxin, 430

[25] S. Kay, Fundamentals Of Statistical Signal Processing, Prentice-Hall, Inc., Upper Saddle River, NJ, USA, 1993. 
[26] R. Chignola, A. Schenetti, E. Chiesa, R. Foroni, S. Sartpris, A. Brendolan, G. Tridente, G. Andrighetto, D. Liberati, Oscillating growth patterns of multicellular tumour spheroids, Cell Proliferation 32 (1) (1999) 39-48. doi: $10.1046 / \mathrm{j} .1365-2184.1999 .3210039 \cdot \mathrm{x}$.

[27] R. Chignola, A. Schenetti, G. Andrighetto, E. Chiesa, R. Foroni, S. Sartoris, G. Tridente, D. Liberati, Forecasting the growth of multicell tumour spheroids: implications for the dynamic growth of solid tumours, Cell Proliferation 33 (4) (2000) 219-229. doi:10.1046/j.1365-2184.2000.00174. $\mathrm{x}$

[28] F. Hirschhaeuser, H. Menne, C. Dittfeld, J. West, W. Mueller-Klieser, L. A. Kunz-Schughart, Multicellular tumor spheroids: an underestimated tool is catching up again, Journal of Biotechnology 148 (1) (2010) 3-15. doi: https://doi.org/10.1016/j.jbiotec.2010.01.012.

[29] I. Lefkovits, H. Waldmann, Limiting dilution analysis of cells in the immune system, Cambridge University Press, Cambridge, 1979.

[30] B. Dennis, J. M. Ponciano, Density-dependent state-space model for population-abundance data with unequal time intervals, Ecology 95 (8) (2014) 2069-2076. doi:10.1890/13-1486.1.

[31] T. B. Schön, A. Wills, B. Ninness, System identification of nonlinear staten space models, Automatica 47 (1) (2011) 39-49. doi:https://doi.org/ $10.1016 / \mathrm{j}$.automatica.2010.10.013

[32] A. C. Charalampidis, G. P. Papavassilopoulos, Development and numerical investigation of new non-linear kalman filter variants, IET Control Theory Applications 5 (10) (2011) 1155-1166. doi:10.1049/iet-cta.2010.0553

[33] Matlab Optimization Toolbox User's Guide, The MathWorks Inc., Natick, MA, 2017. 
[34] A. P. Dempster, N. M. Laird, D. B. Rubin, Maximum likelihood from incomplete data via the em algorithm, JOURNAL OF THE ROYAL STATISTICAL SOCIETY, SERIES B 39 (1) (1977) 1-38.

[35] A. Smirnova, G. Chowell, A primer on stable parameter estimation and forecasting in epidemiology by a problem-oriented regularized least squares algorithm, Infectious Disease Modelling 2 (2) (2017) 268-275. doi:https: //doi.org/10.1016/j.idm.2017.05.004.

[36] A. Kleczkowski, C. A. Gilligan, Parameter estimation and prediction for the course of a single epidemic outbreak of a plant disease, J R Soc Interface 4 (16) (2007) 865-877. doi:10.1098/rsif.2007.1036.

[37] G. Chowell, P. Fenimore, M. Castillo-Garsow, C. Castillo-Chavez, Sars outbreaks in ontario, hong kong and singapore: the role of diagnosis and isolation as a control mechanism, Journal of Theoretical Biology 224 (1) (2003) 1-8. doi:https://doi.org/10.1016/S0022-5193(03)00228-5.

[38] G. Helmlinger, P. A. Netti, H. C. Lichtenbled, R. J. Melder, R. K. Jain, Solid stress inhibits the growth of multicellular tumor spheroids, Nature Biotechnology 15 (1997) 778-783. 


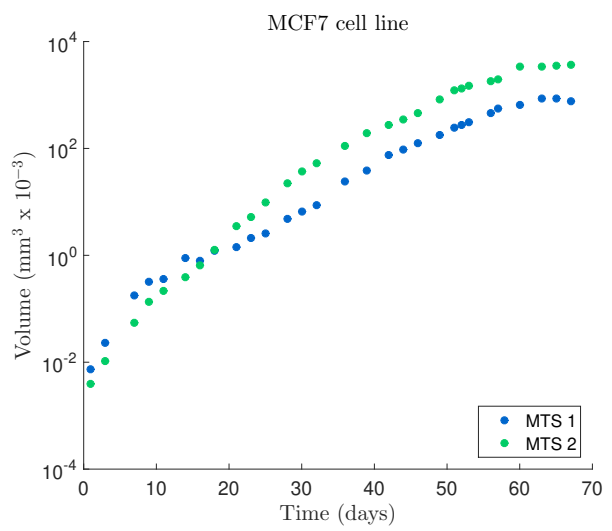

(a) Tumor growth for MCF7 MTS

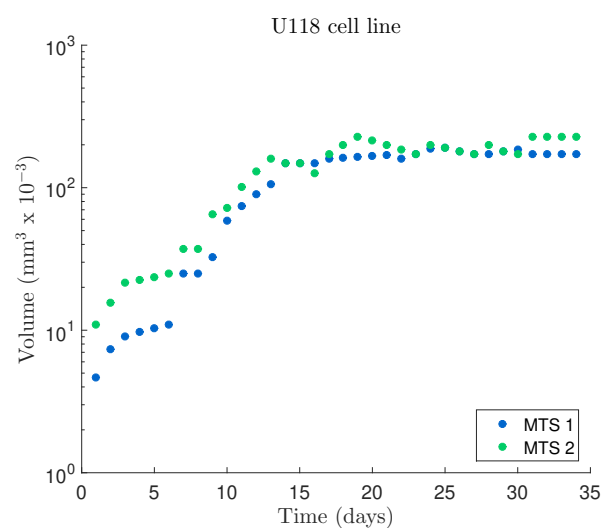

(b) Tumor growth for U118 MTS

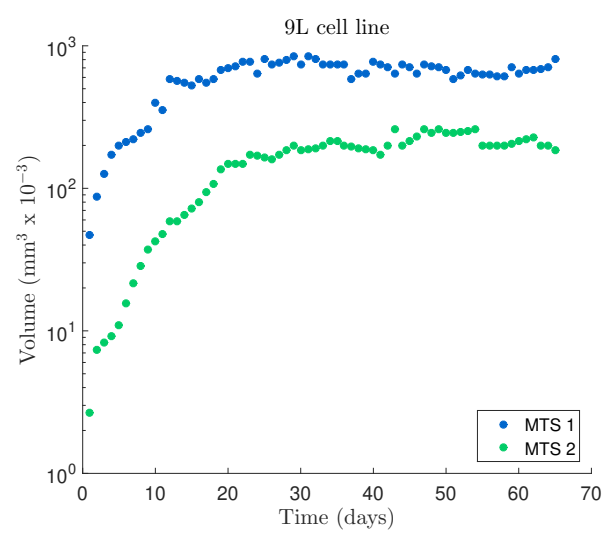

(c) Tumor growth for 9L MTS

Fig. 1. Growth trajectories of multicell tumor spheroids. Representative data on the individual growth of two MCF7, two U118 and two 9L MTS. 

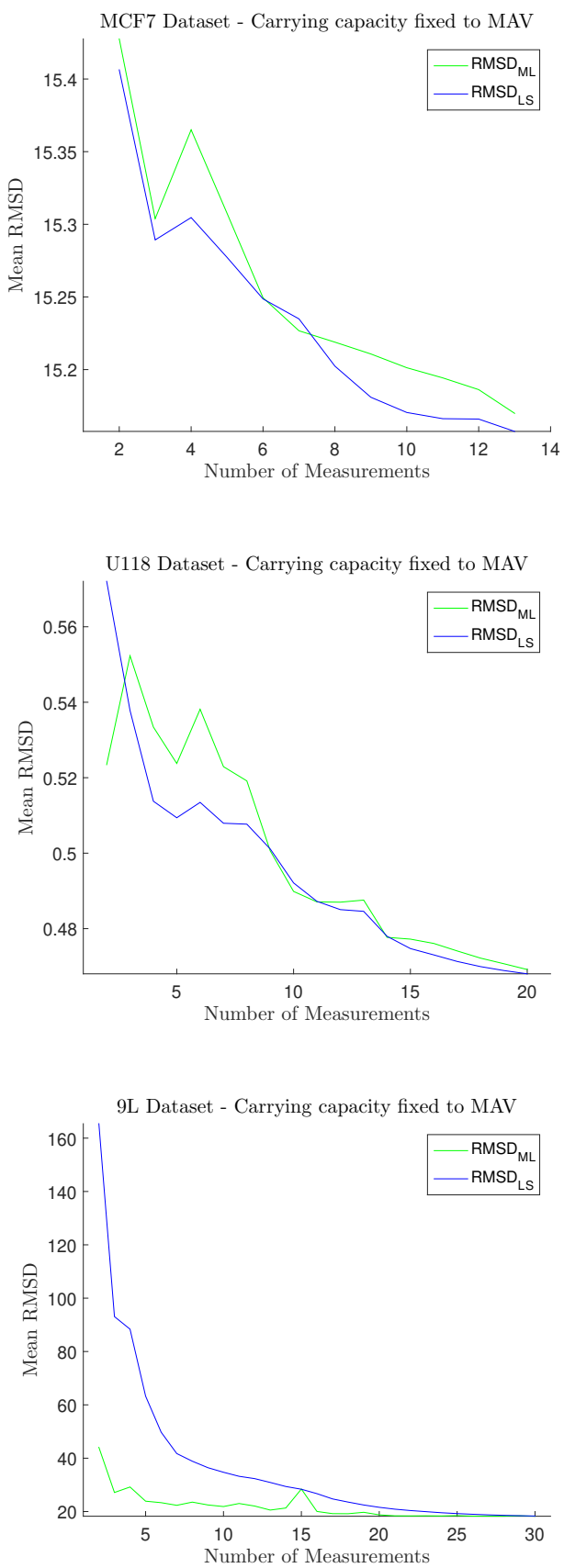

Fig. 2. Mean RMSD for MLE vs NLS $M A V$. 

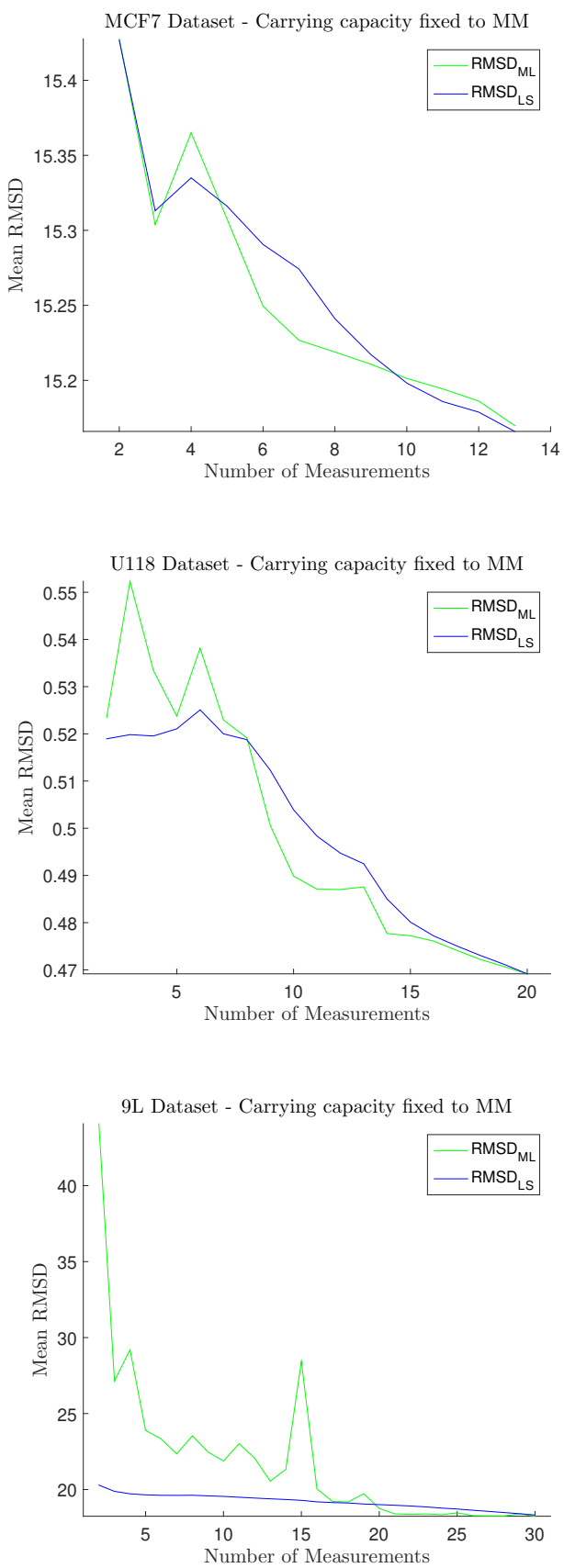

Fig. 3. Mean RMSD for MLE vs $\operatorname{NLS}_{M M}$. 

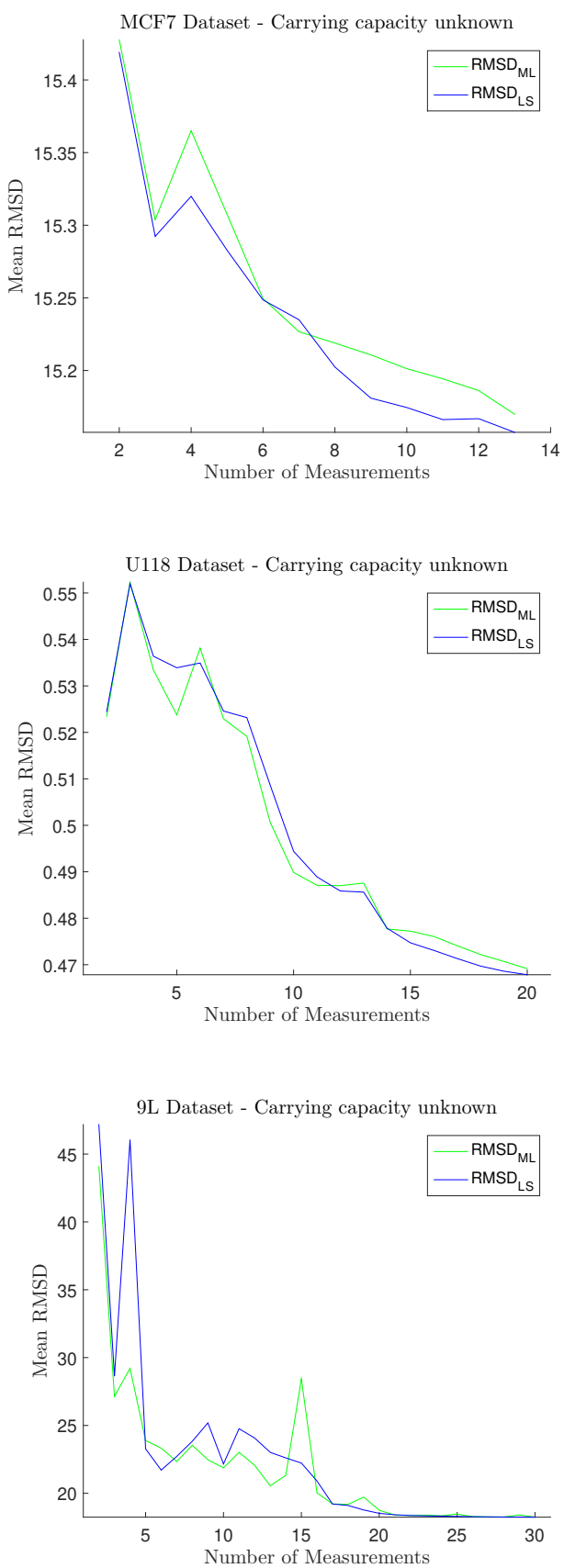

Fig. 4. Mean RMSD for MLE vs $\mathrm{NLS}_{2 p}$. 